\title{
Lymphoepithelial Carcinoma of the Nasal Cavity and Langerhans Cell Histiocytosis: Two Synchronous Neoplasms
}

\author{
Elena Dina ${ }^{1 *}$, Yolanda Escamilla Carpintero ${ }^{2}$ and Carmen María Blazquez Mañá ${ }^{3}$ \\ ${ }^{1}$ Department of Otolaryngology, University Hospital Parc Tauli Sabadell, Spain \\ ${ }^{2}$ Head of the Oncology Unit, Department of Otolaryngology, University Hospital Parc Tauli, Spain \\ ${ }^{3}$ Department of Pathology, University Hospital Parc Tauli Sabadell, Spain
}

Submission: July 12, 2020; Published: August 04, 2020

*Corresponding author: Elena Dina, Otolaryngology Department, Hospital Parc Taulí Parc de Taulí 1, 08208, Sabadell (Barcelona), Spain

Abstract

The authors report a case of a 78-year-old woman with two synchronous neoplasms: Langerhans cell histiocytosis and lymphoepithelial carcinoma of the nasal cavity. The histopathological findings are extremely rare and represent a challenge for the pathologist as the two malignancies coexist within the same tumoral tissue. A review of the literature is presented.

Keywords: Langerhans histiocytosis; Lymphoepithelial carcinoma; Nasal cavity; Synchronous neoplasms; BRAF

Abbreviations : LEC: Lymphoepithelial Carcinoma; NPC: Nasopharyngeal Carcinoma; EBV: Epstein Barr Virus; LCH: Langerhans Cell Histiocytosis; PET/CT: Positron Emission Tomography - Computed Tomography; EBER: Epstein Barr Virus Encoded RNA; ND-LCH: Neurodegenerative Langerhans Cell Histiocytosis

\section{Introduction}

Malignant sinonasal tumors represent less than $1 \%$ of human cancer and approximately $3 \%$ of all malignancies of the head and neck region, with an incidence of 1:100.000. LEC of the nasal cavity and paranasal sinuses has been recently accepted as a distinguishable entity which is topographically distinct, yet histologically like the NPC. Although they are both associated with EBV, there are reports of LEC EBV negative cases [1,2]. LCH is a rare proliferative disorder affecting 5-9:1000.000 children younger than 15 years of age and 5:1000.000 adults annually [35].

\section{Case Report}

The patient was a 78-year-old woman without any known allergies, nonsmoker and who denied alcohol consumption. The medical history included auricular fibrillation, arterial hypertension, diabetes mellitus type II, hypothyroidism secondary to thyroidectomy for nodular goiter. A neurological impairment had appeared nine years previously, with dysarthria, ataxia, dysphagia for liquids with progressive worsening. The initial diagnosis was degenerative ataxia versus multiple system atrophy. The patient rejected any medical treatment for this condition and attended on irregular basis rehabilitation sessions with the speech and swallow therapist. An important weight loss of $20 \mathrm{~kg}$ had occurred for the last four years, with $6 \mathrm{~kg}$ loss during the last six months.

The patient was referred to the hematology department for a $4 \mathrm{~cm}$ right submandibular mass, indurated, non-tender, with gradual enlargement during the last year and with a fine needle aspiration cytology suggestive of LCH.

An adenectomy was performed and the result was metastatic adenopathy of nasopharyngeal carcinoma associated with reactive Langerhans cell proliferation. The patient was referred to the otolaryngology department. She did not present nasal complaints. Subsequent workup included physical exam, biopsy, and imaging. The head and neck examination showed multiple findings. Hypertrophy of the tail of the inferior turbinate and medial bulging of the lateral nasal wall in the inferior meatus were observed during endoscopy in the left nasal fossa. The macroscopic aspect of the nasal mucosa was normal. Biopsies taken from both sites were negative for malignity. The nasopharynx was normal. 


\section{Global Journal of Otolaryngology}

Bulging of the left side of the hard palate was noted (Figure 1a) in the oral cavity. The oral mucosa had normal aspect. The biopsy from this mass was positive for lymphoepithelial carcinoma associated with Langerhans histiocytosis. Reviewing the cervical adenectomy, the same histopathological aspect was observed: lymphoepithelial carcinoma intermingled with Langerhans cell proliferation. Palpation assessment of the neck revealed multiple cervical lymph nodes, the largest on the right side at level IIA. PET/CT scan of the whole body was performed twice (initially and four months later).

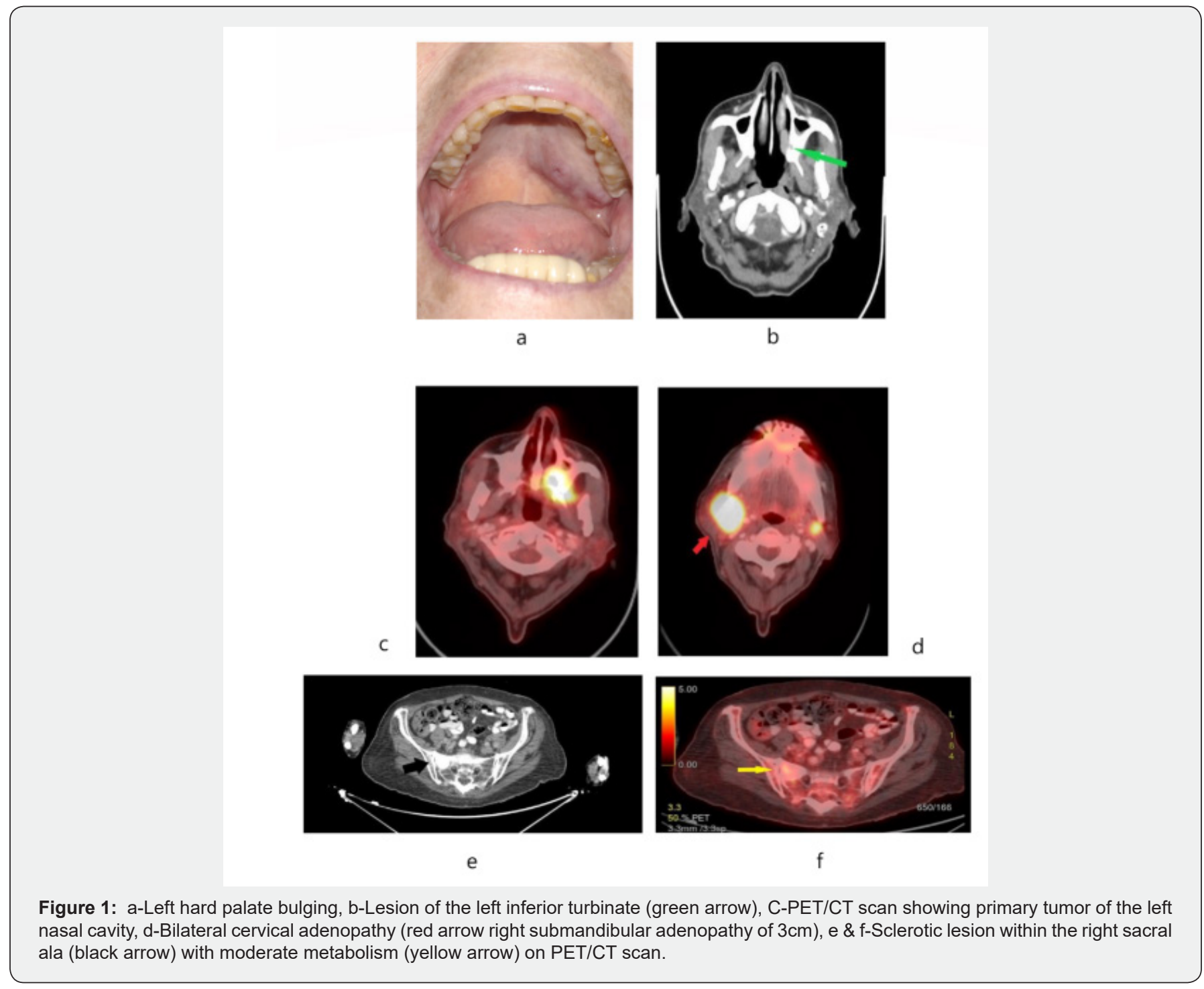

Head and neck imaging showed a hyperdense $18 \mathrm{~mm}$ soft tissue lesion at the tail of the left inferior turbinate (Figure 1b) which also affected the left maxillary bone extending to the hard palate with enlargement of the major palatine foramen and the left pterygopalatine fossa. One hyperintense cervical lesion was noted on the right side at level IIA of $3 \mathrm{~cm}$ diameter and one on the left side of $11 \mathrm{~mm}$ at the same level (Figure $1 \mathrm{~b} \& 1 \mathrm{c}$ ). On the second scan the adenopathy enlarged to $3.5 \mathrm{~cm}$. The nasopharynx was normal. Thorax imaging revealed multiple isolated pulmonary nodules less than one centimeter bilateral, in the upper fields without abnormal metabolism.
Abdominal and pelvic imaging: sclerotic lesion on the right sacral ala of $3 \mathrm{~cm}$ diameter with moderate metabolism (Figure 1e \& 1f). This lesion remained stable. The patient rejected the bone biopsy to rule out a distant metastasis. Serology for Epstein-Barr virus was positive. The patient was evaluated by the multidisciplinary head and neck cancer committee of the authors' institution and was staged as T4N2cM0/ T4N2cM1 (due to the sacral lesion) of LEC of nasal cavity and paranasal sinuses associated with LCH ( $8^{\text {th }}$ edition of TNM classification). Both surgery and chemotherapy were discarded, palliative radiotherapy was reserved in case of worsening of symptoms. 


\section{Global Journal of Otolaryngology}

\section{Discussion}

\section{LEC}

LEC consists of poorly differentiated squamous cell carcinoma or histologically undifferentiated carcinoma accompanied by abundant reactive infiltration of lymphocytes and plasmatic cells. It is associated with EBV infection. However, there are EBV negative cases reported in Europe and USA. The serology does not correlate with the clinical prognose of the disease. It usually affects men between $50-70$ years of age. The nasal septum and nasal cavity are the most affected sites. It is a radiosensitive neoplasm. Only 15\% of LEC cases presents with lymphadenopathy in comparison with $85 \%$ of NPC cases [2,6-8].

LEC is characterized by a submucosal neoplastic proliferation associated by a nonneoplastic lymphoplasmacytic infiltrate (less abundant than in NPC). Neoplastic cells have large round to oval nuclei with one or more nucleoli and abundant amphophilic cytoplasm. Nuclear polymorphism can be observed. The mitotic activity usually is not increased. Neoplastic cells are cytokeratin positive and usually the EBER staining is intensely positive. P16 staining, melanoma-related markers, hematolymphoid markers and neuroendocrine/ neuroectoderm markers are absent [1].

Differential diagnosis of sinonasal LEC is made mainly with the sinonasal undifferentiated carcinoma, non- Hodgkin lymphoma and nasopharyngeal carcinoma. There are few cases published of lymphoepithelial carcinoma of the nasal cavity and the paranasal sinuses. Takakura et al. presented in 2018 a review of the previously 9 reported cases of sinonasal lymphoepithelial carcinoma between 1980-2018. There are only two case series published with 33 patients registered: Zong et al. [6] 20 patients and Jeng et al. [7] 13 patients. To avoid confusion, it is important to mention that LEC was previously named nasopharyngeal-type undifferentiated carcinoma of the nasal cavity and paranasal sinuses $[2,6,7]$.

\section{LCH}

Histiocytosis are disorders characterized by accumulation of macrophages, dendritic cells, monocyte-derived cells in tissues and organs. There are over 100 subtypes described. The revised classification published by Emile et al. [9] in 2016 consists of five groups: Langerhans-related, cutaneous and mucocutaneous, malignant histiocytosis, Rosai-Dorfman disease, hemophagocytic lymph histiocytosis and macrophage activation syndrome [9]. LCH is a clonal proliferation of CD1a+ and CD207+ cells. The clonality itself does not imply a neoplastic process. The BRAF V600E mutation discovered in more than half of the patients is not specific for LCH but it is a neoplastic disease [2].

The diagnosis of $\mathrm{LCH}$ requires the detection of Langerhans cell markers. Detection of CD1a and CD207 (langerina) expression has replaced the detection of Birbeck granules and permits differential pathological diagnosis with other entities like indeterminate cell histiocytosis (CD207 immunostaining negative) and RosaiDorfman disease. Langerhans cells are often accompanied by eosinophils and multinucleated giant cells. Although the mitotic activity might be high, the diagnosis of malignant histiocytosis cannot be made in the absence of major nuclear atypia and clinical evidence of rapid tumor progression [9].

Association between LCH and neoplasms has been reported. Ma et al. [10] published in 2018 a case series of 132 consecutive adult patients with LCH (1990-2015). One third of the patients had another neoplastic disease: a solid tumor (74\%), a lymphoma (17\%) or a hematologic malignancy (9\%). The most frequent solid tumor was lung cancer followed by breast and colorectal cancer. Follicular and Hodgkin lymphoma were the most common in the lymphoma group and acute myeloid leukemia was the most common hematologic malignancy. Mutations within BRAF/ERK genes and / or use of carcinogenic agents like etoposide may be implicated in the tumor genesis [10].

\section{The case presented}

The histopathologic examination of both biopsies is similar: a unique pattern with clusters of lymphoepithelial carcinoma consisting of epithelial cell nests and presence of abundant histiocytic cells closely intermingled with epithelial component, as a network encompassing epithelial cells (Figure 2). Carcinomatous cells expressed immunohistochemical markers of squamous origin ( $\mathrm{p} 40$ ), negative expressions for $\mathrm{p} 16$ and intense and diffuse nuclear pattern positivity for EBER. The histiocytic component was positive for S-100 protein, CD1a and CD207, confirming the diagnosis of Langerhans cell histiocytosis (Figure 3a \& 3b).

Determination of the mutational status of the BRAF gene was performed for both epithelial and histiocytic components, confirming the presence of the V600E mutation of the BRAF in the Langerhans cell component and not in the epithelial component (Figure 4). This finding confirms two synchronous neoplasms within the same tumor. The neurologic condition of the patient is compatible with ND-LCH. This rare condition has miscellaneous manifestations from subtle to serious neurologic impairment (e.g. ataxia, spastic quadriparesis, intellectual disability, psychiatric symptoms) [11]. Characteristic brain MRI changes are described for ND-LCH and elevated osteopontina in the cerebrospinal fluid helps differentiating ND-LCH from other neurodegenerative disorders [11,12]. Unfortunately, a complete study in the case presented was not made. Nevertheless, the association between the neurologic symptoms and the stable sacral bone lesion might indicate a previous long standing LCH.

\section{Conclusion}

Further research is needed to clarify the relationship between the LEC and the LCH (if one can induce the other or if there is a common initial trigger for both malignancies). The clinical case reported is exceedingly rare, probably the first reported till now. 

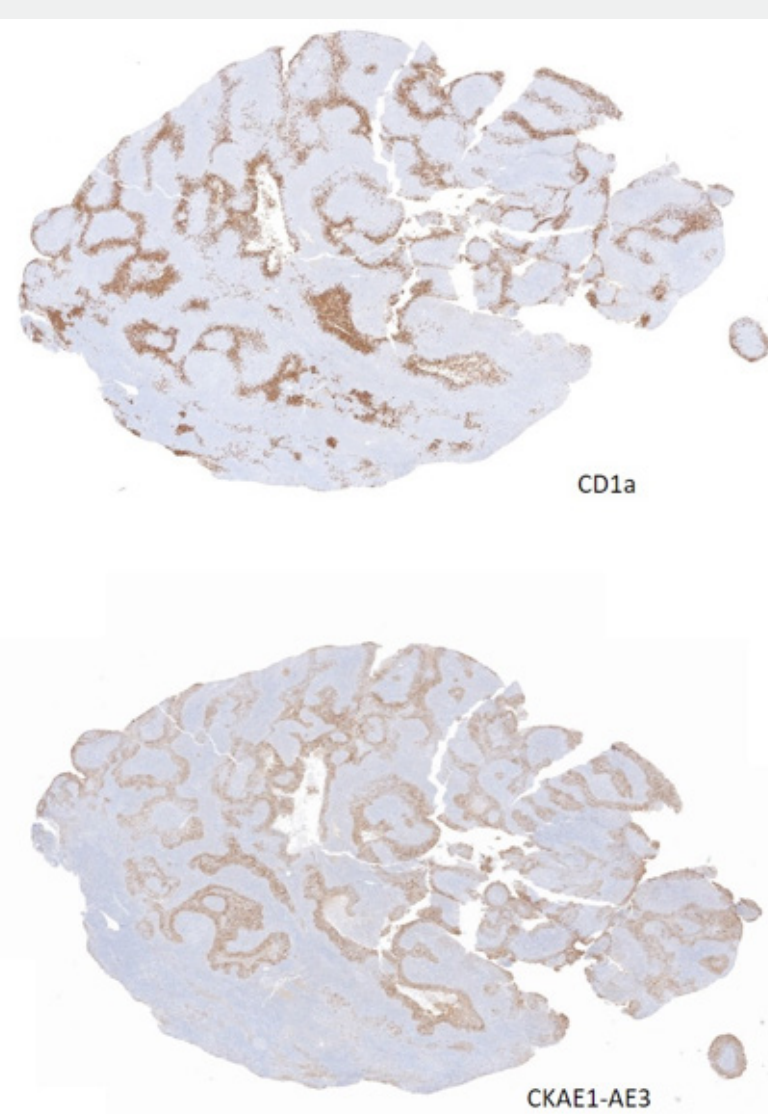

Figure 2: Pattern of expressions of cytokeratin AE1/AE3 and CD1a showing Langerhans cells making a network that involve epithelial cells.
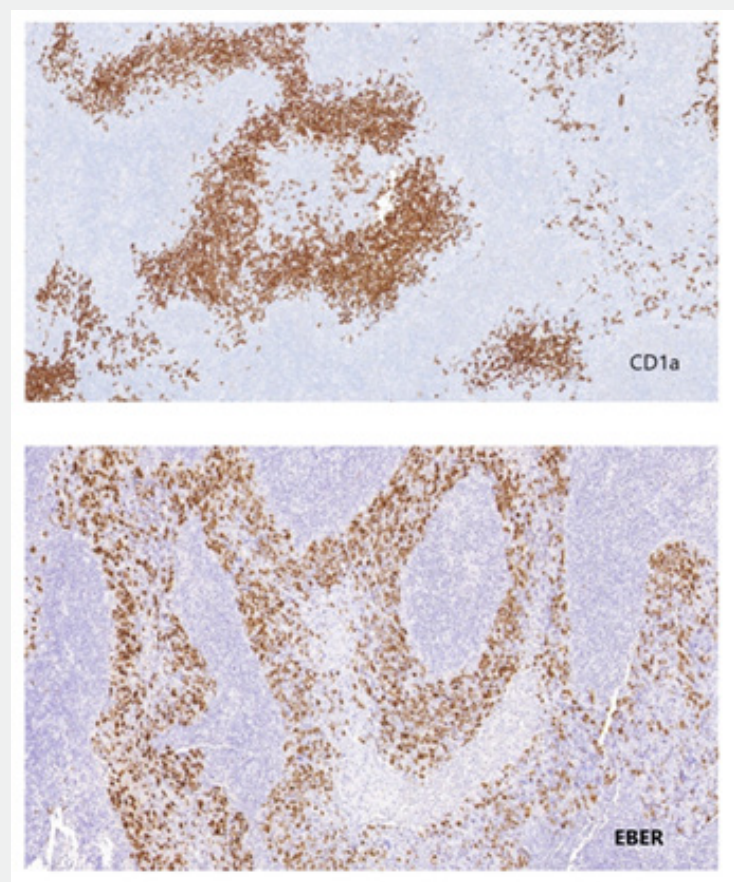

Figure 3a \& 3b: Expression of CD1a in Langerhans cells and positivity for EBER staining. The images show the close relationship between both cell types. 


\section{Global Journal of Otolaryngology}

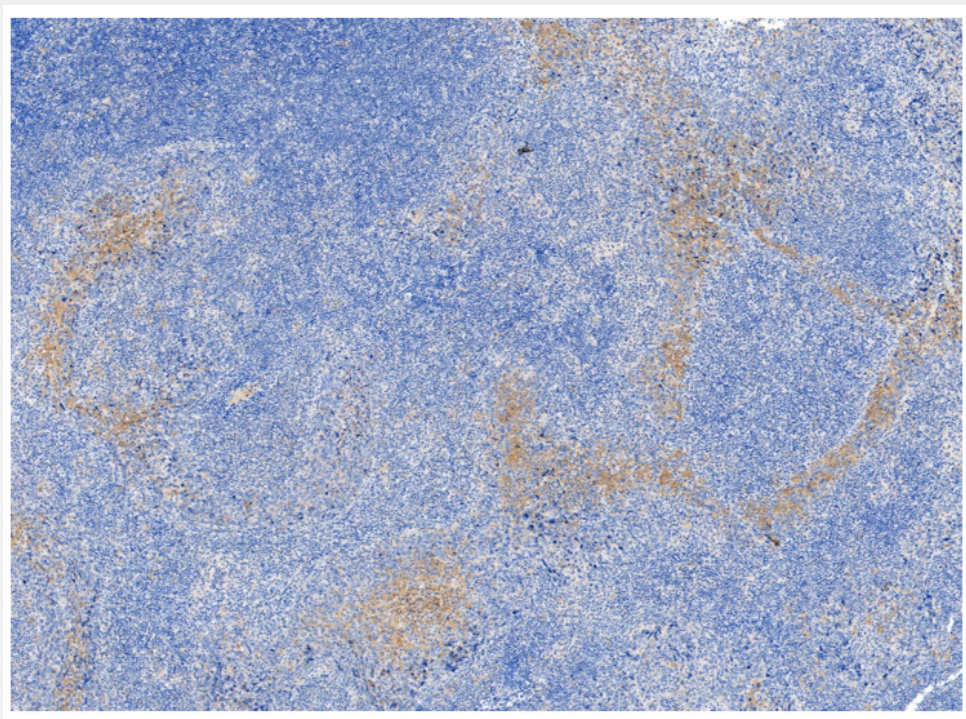

Figure 4: Expressions of BRAF (clone VE1, Ventana) in the histiocytic component, showing the mutated status of BRAF in Langerhans cells.

\section{Consent}

Written informed consent was obtained from the patient for publication of this case report and the accompanying images.

\section{References}

1. Bruce W (2014) Lymphoepithelial-Like Carcinomas of the Head and Neck. Semin Diagn Pathol 32: 74-86.

2. Takakura H, Tachino H, Fujisaka M, Nakajima T, Yamagishi K, et al. (2018) Lymphoepithelial carcinoma of the maxillary sinus: A case report and review of the literature. Medicine (Baltimore) 97(28): e11371.

3. Krooks J, Minkov M, Weatherall AG (2018) Langerhans cell histiocytosis in children: History, classification, pathobiology, clinical manifestations, and prognosis. J Am Acad Dermatol 78(6): 1035-1044.

4. Monsereenusorn C, Galindo RC (2015) Clinical Characteristics and Treatment of Langerhans Cell Histiocytosis. Hematol Oncol Clin North Am 29(5): 853-873.

5. Nakamine H, Yamakawa M, Yoshino T, Fukumoto T, Enomoto Y, et al. (2016) Langerhans Cell Histiocytosis and Langerhans Cell Sarcoma: Current Understanding and Differential Diagnosis. J Clin Exp Hematop 56(2): 109-118.

6. Zong Y, Liu K, Zhong B, Chen G, Wu W (2001) Epstein-Barr virus infection of sinonasal lymphoepithelial carcinoma in Guangzhou. Chin Med J (Engl) 114(2):132-136.

7. Jeng YM, Sung MT, Fang CL, Huang HY, Mao TL, et al. (2002) Sinonasal undifferentiated carcinoma and nasopharyngeal-type undifferentiated carcinoma: two clinically, biologically, and histopathologically distinct entities. Am J Surg Pathol 26(3): 371-376.

8. Ho FC, Tham IW, Earnest A, Lee KM, Lu JJ (2012) Patterns of regional lymph node metastasis of nasopharyngeal carcinoma: a meta-analysis of clinical evidence. BMC Cancer 12: 98.

9. Emile JF, Abla O, Fraitag S, Horne A, Haroche J, et al. (2016) Revised classification of histiocytoses and neoplasms of the macrophagedendritic cell lineages. Blood 127(22): 2672-2681.

10. Ma J, Laird JH, Chau KW, Chelius MR, Lok BH, et al. (2019) Langerhans cell histiocytosis in adults is associated with a high prevalence of hematologic and solid malignancies. Cancer Med 8(1): 58-66.

11. Sieni E, Barba C, Mortilla M, Savelli S, Grisotto L, et al. (2015) Early Diagnosis and Monitoring of Neurodegenerative Langerhans Cell Histiocytosis. PLoS One 10(7): e0131635.

12. McClain KL, Picarsic J, Chakraborty R, Zinn D, Lin H, et al. (2018) CNS Langerhans cell histiocytosis: Common hematopoietic origin for $\mathrm{LCH}-$ associated neurodegeneration and mass lesions. Cancer 124(12): 2607-2620. 
This work is licensed under Creative (C) (i) Commons Attribution 4.0 License BY DOI: 10.19080/GJO.2020.23.556103
Your next submission with Juniper Publishers will reach you the below assets

- Quality Editorial service

- Swift Peer Review

- Reprints availability

- E-prints Service

- Manuscript Podcast for convenient understanding

- Global attainment for your research

- Manuscript accessibility in different formats

( Pdf, E-pub, Full Text, Audio)

- Unceasing customer service

Track the below URL for one-step submission https://juniperpublishers.com/online-submission.php 